\section{CÁNCER Y FERTILIDAD}

\author{
Begoña Bermejo \\ Hospital Clínico Universitario de Valencia \\ begobermejo@terra.es \\ Blanca Navarro \\ Hospital Clínico Universitario de Valencia \\ blancanav@hotmail.com \\ Ana Lluch \\ Hospital Clínico Universitario de Valencia \\ ana.lluch@uv.es
}

Cómo citar este artículo/Citation: Bermejo, B.; Navarro, B.; Lluch, A. (2015). “Cáncer y fertilidad”. Arbor, 191 (773): a239. doi: http://dx.doi.org/10.3989/arbor.2015.773n3009

Recibido: 9 diciembre 2014. Aceptado: 24 abril 2015.

RESUMEN: Los cambios sociológicos de las últimas décadas han llevado a que el inicio de la maternidad se retrase de forma considerable. Por otra parte, el diagnóstico temprano de algunos cánceres como el de mama o el de cérvix y el progreso en los tratamientos oncológicos han conseguido tasas de curación muy favorables. Todo ello lleva a que la mujer, que sobrevive cada vez más a esta enfermedad, desee completar su deseo genésico que se va a ver mermado muchas veces por los tratamientos recibidos. Tener hijos es parte importante de la vida de muchas personas, y los médicos (oncólogos, cirujanos, ginecólogos, etc.) deben informar adecuadamente al paciente sobre los riesgos que su proceso conlleva sobre la fertilidad y conocer y ofrecer las diferentes opciones de preservarla. Durante este artículo nos ceñiremos a hablar de la fertilidad en la mujer, cómo se afecta, y cómo protegerla.

PALABRAS CLAVE: criopreservación; estimulación ovárica; quimioprofilaxis; transposición ovárica.

\section{CANCER AND FERTILITY}

Copyright: (C) 2015 CSIC. Este es un artículo de acceso abierto distribuido bajo los términos de la licencia Creative Commons Attribution-Non Commercial (by-nc) Spain 3.0.

ABSTRACT: The sociological changes occurring in recent decades have led to women having their first child much later than in the past. On the other hand, the early diagnosis of some tumors, such as breast or cervix, and the advances made in oncological treatments have achieved very favorable cure rates. All these aspects lead to the fact that women, whose chances of surviving this disease are improving all the time, wish to fulfill their wish to have children, which is going to be frequently lessened due to the treatments received. Having children is an important part of life for many people, and clinicians (oncologists, surgeons, gynecologists, etc.) must inform the patient properly about the risks that the process entails regarding fertility, as well as explaining the different options to preserve it. In this article, we will discuss fertility in women only, how it is affected, and how to protect it.

KEYWORDS: chemoprophylaxis; cryopreservation; ovarian stimulation; ovarian transposition. 


\section{INTRODUCCIÓN}

Los cambios sociológicos de las últimas décadas han llevado a que el inicio de la maternidad se retrase de forma considerable. Por otra parte el diagnóstico temprano de algunos cánceres como el de mama o el de cérvix y el progreso en los tratamientos oncológicos han conseguido unas tasas de curación muy favorables. Todo ello lleva a que la mujer, que sobrevive cada vez más a esta enfermedad, desee completar su deseo genésico que se va a ver mermado muchas veces por los tratamientos recibidos. Alrededor de un $4 \%$ de los diagnósticos de cáncer en EEUU se dan en personas menores de 35 años. Los diagnósticos más frecuentes en estas edades son: cáncer de mama, melanoma, cáncer de cérvix, linfoma no Hodgkin, y leucemia (Lee et al., 2006; Siegel et al., 2014).En los últimos años se han producido avances importantes en la medicina reproductiva y como resultado se ha objetivado un aumento en la eficacia de las técnicas de preservación de la fertilidad y en la seguridad de dichas técnicas.

Tener hijos es parte importante de la vida de muchas personas y los médicos (oncólogos, cirujanos, ginecólogos, etc.) deben informar adecuadamente al paciente sobre los riesgos que su proceso conlleva sobre la fertilidad y conocer y ofrecer las diferentes opciones de preservarla. Durante este capítulo nos ceñiremos únicamente a hablar de la fertilidad en la mujer, cómo se afecta y cómo protegerla. Hablar de la fertilidad en los hombres o de técnicas de preservación de la fertilidad en niños no es objeto de este capítulo.

\section{ALGUNOS CONCEPTOS BÁSICOS}

Antes de abordar las diferentes maneras y técnicas de preservación de la fertilidad vale la pena recordar algunos conceptos sobre la anatomía y la fisiología reproductora de la mujer.

Durante el desarrollo femenino, al llegar a la pubertad se inicia la actividad hormonal a nivel central en el denominado eje hipotálamo-hipofisario, situado en la base del cráneo. Este proceso estimula el ovario con la consiguiente producción hormonal y de gametos, llevando al inicio del periodo fértil en la mujer. El proceso completo consta de las siguientes fases:

1. Fase pre-gonadotrópica. Durante el proceso de formación del embrión femenino, tiene lugar la formación de las células germinales en los ovarios hasta un número determinado (alrededor de 6 millones), los Ilamados folículos primordiales. Estos se transforman en folículos antrales que responderán a las hormonas hipofisarias (las gonadotropinas).
2. Fase dependiente de gonadotropinas, una vez alcanzada la pubertad. Se puede dividir en dos fases

a. Fase folicular. Se selecciona y divide un solo folículo, que madura expulsando el óvulo a la trompa de Falopio donde permanecerá unas horas y puede ser fecundado. Por otra parte, con el estímulo de las hormonas secretadas por el ovario (estradiol y progesterona) se producen cambios proliferativos y secretores en el endometrio quedando preparado para la implantación del embrión en caso de producirse la fecundación.

b. Fase lútea. El folículo que ha liberado el óvulo en el ovario, se convierte en el cuerpo lúteo, que secretará la progesterona. Si no se produce la fecundación tras 14 días degenera y disminuye la producción hormonal lo que conlleva la desestructuración del endometrio que se desprende produciendo la menstruación.

Así, el ciclo menstrual ovárico abarca todos los procesos que ocurren entre dos menstruaciones. El proceso se inicia en el ovario independientemente de la secreción hormonal de la hipófisis (a nivel central) y que consiste en que los folículos primordiales inicien su maduración. Posteriormente con la intervención de las hormonas hipofisarias se selecciona un óvulo que es el que madurará, y el resto de folículos que iniciaron la maduración entran en muerte celular (apoptosis).

Dentro de la fase pre-gonadotrópica es importante entender que la mujer nace ya con un número determinado de precursores de óvulos que se van perdiendo desde el nacimiento, de modo que finalmente en la vida de la mujer habrá alrededor de 400 ovulaciones, el resto de precursores serán degradados. Hablamos por tanto de una reserva ovárica que se va consumiendo hasta llegar a la menopausia. Entre los 16 y los 30 años se encuentra el periodo más fértil de la mujer. Según la reserva ovárica que se tenga antes de iniciar los tratamientos oncológicos existirá mayor o menor riesgo de fallo gonadal.

\section{EFECTOS DE LOS TRATAMIENTOS DEL CÁNCER SO- BRE LA FUNCIÓN REPRODUCTIVA}

El tratamiento del cáncer a menudo pone en peligro la fertilidad. La infertilidad se define como la incapacidad de concebir tras haberlo intentado durante un año sin métodos contraceptivos. Entre el arsenal de armas terapéuticas contra el cáncer, no todas tienen el mismo efecto sobre la función reproductiva. Repasaremos brevemente cómo y en qué medida afectan estos tratamientos. 


\subsection{Quimioterapia}

La quimioterapia afecta a lo que hemos llamado previamente reserva ovárica (el número de folículos primordiales que quedan desde el nacimiento). Conlleva la pérdida de folículos al promover la muerte celular. Los efectos de la quimioterapia en la función ovárica incluyen amenorrea temporal por pérdida de los folículos ováricos en desarrollo o amenorrea permanente por pérdida de los folículos restantes. Los efectos varían según el fármaco utilizado y la edad de la paciente. Agentes alquilantes como la ciclofosfamida son muy gonadotóxicos (Kishk y Mohammed Ali, 2013) ya que no son ciclo específicos y dañan los folículos primordiales remanentes aumentando el riesgo de fallo ovárico. Esto no ocurre con otros fármacos como el metotrexate o el 5-fluorouracilo que sí son ciclo específicos y no dañan la reserva ovárica. En la Tabla 1 se clasifican de forma sencilla diferentes quimioterápicos según su potencialidad en producir infertilidad (Sonmezer y Oktay, 2006).

\subsection{Radioterapia}

Otra estrategia terapéutica ampliamente utilizada en cáncer es la radioterapia. Este tratamiento usa radiación ionizante para destruir células malignas. También lesiona células del área que recibe tratamiento, aunque este daño se intenta limitar al máximo. Según en qué zona del cuerpo humano se administre y a qué dosis supondrá mayor o menor riesgo para la fertilidad. Así, los tratamientos que más afectarán la fertilidad son:

- Irradiación del abdomen o la pelvis. A partir de 6 Gy en mujeres adultas y de 10 Gy en adolescentes que han alcanzado la pubertad.

- Irradiación cerebral total (más de 40 Gy) o del eje hipotálamo-hipofisario.

- Irradiación corporal total.

\subsection{Hormonoterapia}

En cáncer de mama, y también en algunos otros procesos neoplasicos, se utilizan otros tratamientos sistémicos que no son quimioterapia, como la hormonoterapia. El tamoxifeno es el tratamiento estándar en mujeres en edad fértil operadas de cáncer de mama con expresión de receptores hormonales. Este fármaco, por si mismo tiene un bajo riesgo de fallo ovárico, siendo edad dependiente: en menores de 45 años no aumenta el riesgo y en mayores de 45 años aumenta el riesgo de fallo ovárico un $10 \%$ respecto a las mujeres no tratadas. La incidencia de amenorrea sin embargo, aumenta especialmente cuando se usa tras la quimioterapia. Además es teratógeno, por lo que se debe evitar el embarazo bajo este tratamiento. Los análogos de las gonadotropinas, asociados a tratamientos hormonales, causan un reposo ovárico que cursa con amenorrea pero este es reversible tras su retirada.

\subsection{Terapias dirigidas y tratamientos biológicos}

Hay poca información sobre el impacto que estas nuevas terapias tienen sobre la fertilidad. De hecho, en general se desconoce el efecto de fármacos como cetuximab, erlotinib e imatinib y otros sobre la función reproductiva, además la mayoría de ellos se emplean como tratamiento de la enfermedad metastásica, situación en la cual la fertilidad y el deseo reproductivo deja de ser relevante. En el caso de bevacizumab sí se dispone de información. En un estudio se observó fallo ovárico en un $34 \%$ de las mujeres que lo recibían como tratamiento para el cáncer de colon comparado con el fallo ovárico observado en tan solo el $2 \%$ de las mujeres que llevaba el mismo régimen de tratamiento quimioterápico pero sin bevacizumab. Posteriormente solo un quinto de esas mujeres recuperaron la función ovárica. En el caso de inhibidores tiroisin-kinasa como imatinib, su uso más extendido es como tratamiento de la leucemia mieloide crónica, parece que

Tabla 1. Agentes quimioterápicos según su potencialidad en producir infertilidad

\begin{tabular}{|l|l|l|}
\hline Alto riesgo & Riesgo intermedio & Bajo riesgo \\
\hline Ciclofosfamida & Cisplatino & Fluorouracilo \\
Melfalán & Adriamicina & Vincristina \\
Busulfán & Paclitaxel & Bleomicina \\
Mostaza nitrogenada & & Actinomicina D \\
Clorambucilo & & \\
Procarbacina & & \\
\hline
\end{tabular}


existe un efecto adverso sobre la función ovárica. Por otra parte hay evidencia en modelos animales de su teratogenicidad, por lo que se debe desaconsejar el embarazo bajo este tratamiento. Se conocen algunos casos de mujeres que han concebido bajo tratamiento con imatinib en los que hay una mayor proporción de malformaciones graves durante la gestación, algunas de ellas incompatibles con la vida (Zamah et al., 2011). Respecto a trastuzumab, fármaco que se utiliza en el tratamiento del cáncer de mama con sobreexpresión de la proteína Her2, en los estudios adyuvantes que compararon quimioterapia y quimioterapia más trastuzumab, la adición del anticuerpo no se tradujo en un incremento de los trastornos menstruales. En otro reciente estudio, con el uso de la combinación de paclitaxel y trastuzumab como tratamiento adyuvante en pacientes premenopáusicas, la tasa de amenorrea parece ser menor que la observada en regímenes más clásicos que incluyen agentes alquilantes (Ruddy et al., 2015). Trastuzumab, por tanto, probablemente es poco o nada gonadotóxico.

\section{RIESGO DE INFERTILIDAD EN LOS TRATAMIENTOS ONCOLÓGICOS}

En las mujeres, la fertilidad puede verse comprometida por cualquier tratamiento que disminuya el número de folículos primordiales, afecte al balance hormonal, interfiera con el funcionamiento de los ovarios, las trompas de Falopio, el útero o el cérvix.Como ya se ha mencionado, los efectos de la quimioterapia dependen de qué fármaco y a que dosis se esté utilizando y los de la radioterapia dependen de qué zona, y a qué dosis se irradie. Otros factores que modifican el riesgo de infertilidad son la propia enfermedad, la edad y el estado de fertilidad previo al tratamiento de la paciente.Un aspecto relevante a tener en cuenta es que la fertilidad en la mujer puede estar comprometida con independencia del mantenimiento o no de la menstruación. Cualquier descenso en la reserva ovárica puede disminuir las posibilidades de concepción y adelantar la menopausia. Así, incluso en las mujeres fértiles tras el tratamiento, la fertilidad puede verse acortada por una menopausia prematura.

Se ha recogido información sobre el riesgo de amenorrea en mujeres tratadas con diferentes tratamientos.

1. Alto riesgo de amenorrea (70\%):

a. Trasplante de progenitores hematopoyéticos con ciclofosfamida junto con irradiación corporal total, o ciclofosfamida junto con busulfán. b. Cualquier agente alquilante: busulfán, ciclofosfamida, ifosfamida, lomustina, melfalán, procarbacina.

c. Radioterapia externa en campos que incluyan los ovarios.

d. Radioterapia cerebral asociada o no a temozolamida o BCNU.

e. Esquemas de quimioterapia con combinaciones de ciclofosfamida, metrotexate, fluoracilo, doxorrubicina y epirrubicina, en el caso de mujeres de edad mayor o igual a 40 años que reciben 6 ciclos de estos tratamientos.

2. Riesgo intermedio de amenorrea (entre 30-70\%)

a. Esquemas de quimioterapia con combinaciones de ciclofosfamida, metrotexate, fluorouracilo, doxorrubicina y epirrubicina, en mujeres de entre 30 y 39 años que recibe 6 ciclos de estos tratamientos.

b. Esquemas con doxorrubicina y ciclofosfamida, 4 ciclos, como el usado en la adyuvancia de cáncer de mama, en mujeres de 40 años o más.

c. Anticuerpos monoclonales como bevacizumab.

d. Protocolos que contengan cisplatino.

3. Bajo riesgo de amenorrea (menos de un $30 \%$ ).

a. 4 ciclos de esquemas con doxorrubicina y ciclofosfamida como el usado en la adyuvancia del cáncer de mama en mujeres de menos de 40 años.

b. 6 ciclos de tratamiento con esquemas de quimioterapia con combinaciones de ciclofosfamida, metrotexate, fluorouracilo, doxorrubicina y epirrubicina, en mujeres de menos de 30 años.

c. Esquemas usados en linfomas como CHOP, ABVD, CVP.

d. Antraciclinas y citarabina, usados en leucemia mieloide aguda.

4. Se consideran prácticamente sin riesgo fármacos como vincristina, metotrexate y fluorouracilo y el tratamiento con yodo radioactivo, usado en cáncer de tiroides.

5. En otros casos se desconoce el riesgo como ocurre con fármacos como oxaliplatino, taxanos, irinotecan, anticuerpos monoclonales como trastuzumab, cetuximab, etc., o fármacos inhibidores de tirosinkinasas como erlotinib, imatinib, etc. 
Además de esto no hay que olvidar otros factores mencionados previamente como la reserva ovárica previa al tratamiento, la edad, etc.

\section{ESTRATEGIAS DE PRESERVACIÓN DE FERTILIDAD}

Se disponen de varias estrategias para preservar la fertilidad previa al inicio de los tratamientos oncológicos. Hay que tener en cuenta que la eficacia de los diferentes métodos de preservación de la fertilidad se ve comprometida por la edad. Además, la estrategia a utilizar se verá condicionada por el diagnóstico, el tipo de tratamiento, si la paciente tiene o no pareja, por el riesgo de metástasis ováricas, etc.

A continuación, explicaremos brevemente las siguientes técnicas: quimioprofilaxis, criopreservación de ovocitos, criopreservación de embriones, criopreservación del tejido ovárico, transposición ovárica y cirugías ginecológicas conservadoras(Loren et al., 2013).

\subsection{Quimioprofilaxis}

Es una estrategia que pretende conseguir la supresión ovárica mediante el uso de agonistas o antagonistas de las gonadotropinas, para proteger los ovocitos durante el tratamiento.En humanos, el uso de estos tratamientos como método para preservar la fertilidad es controvertido (Blumenfeld, 2007; Del Mastro et al., 2011). Se ha demostrado su efecto protector en ratas, pero no está tan claro su efecto en humanos, ya que existen diversos estudios con resultados contradictorios. La mayoría de estos estudios tienen un número pequeño de pacientes y no están comparados con brazos control. Otro problema de muchos de estos estudios es que miden la recuperación de la menstruación como marcador de recuperación de la fertilidad y hoy sabemos que esto no es así, el hecho de que la mujer vuelva a menstruar no implica que haya recuperado la fertilidad. Además, la supresión ovárica completa no se consigue hasta que pasan varias semanas tras el inicio de la administración de estos tratamientos.

Por otra parte, pequeños estudios observacionales sugieren que los anticonceptivos orales administrados durante la quimioterapia podrían ayudar a preservar la función ovárica.Hay en marcha estudios con mejores diseños que pueden arrojar más información al respecto, pero por ahora, debido a la falta de información de calidad no se recomienda esta estrategia como una opción estándar de preservación de la fertilidad.

\subsection{Estimulación ovárica}

La estimulación ovárica se utiliza previa a la recogida de ovocitos, para su criopreservación o para su fecundación y posterior criopreservación de embriones. Se puede hacer con antagonistas de las gonadotropinas que permiten el comienzo precoz de la estimulación independientemente del momento del ciclo en el que se encuentra la paciente. Se administran dosis diarias hasta conseguir niveles basales de estradiol. En ese momento se pueden recuperar ovocitos inmaduros o el ovocito que madura en un ciclo natural. Otra opción es administrar inhibidores de aromatasa como letrozol, que induce la supresión de los niveles de estrógeno circulantes, ya sea solo o en combinación con FSH.

Con el uso de agonistas de las gonadotropinas se consigue disminuir los niveles de estradiol postcaptación ovárica y disminuye el riesgo del síndrome de hiperestimulación ovárica. No obstante, con los protocolos de antagonistas de las gonadotropinas se consigue disminuir la duración del tratamiento a 10 días, de modo que el retraso en el inicio de tratamiento para el cáncer es el menor posible. Otra opción es recuperar ovocitos inmaduros, madurarlos in vitro y una vez madurados vitrificarlos. Con ello se podría prescindir de la estimulación ovárica y no dilatar el tiempo al inicio del tratamiento. Sin embargo, las series de casos presentadas son muy limitadas y aun hay una alta tasa de abortos, por lo que esta técnica debe ser considerada experimental.

\subsection{Criopreservación de ovocitos}

Requieren técnicas de estimulación ovárica y posterior recolección de los ovocitos bajo sedación, por vía transvaginal guiada por ecografía. Posteriormente, estos ovocitos no fertilizados son criopreservados. Más adelante, estos ovocitos serán fertilizados in vitro. Este método es una opción para las pacientes que no tienen pareja y prefieren no usar un donante de semen, o que tienen objeciones éticas o religiosas a la criopreservación de embriones. Por otra parte los ovocitos no fecundados tienen mayor tendencia a dañarse que los embriones durante las técnicas de criopreservación y las tasas de embarazo pueden ser algo menores.Si bien, con la nueva técnica de vitrificación de óvulos se consiguió mejorar estos resultados. La vitrificación es una nueva técnica de criopreservación para ovocitos que consigue criopreservar ovocitos con éxito, cosa que no se ha conseguido con la congelación lenta (Cobo et al., 2008; Cobo et al., 2013). Una vez se han extraído los óvulos, estos se trasladan al laboratorio de embriología donde se estudian y proce- 
san en condiciones de máxima esterilidad, y aquellos que sean aptos, se conservarán en nitrógeno líquido a -196 C para poder disponer de ellos una vez la paciente haya superado su enfermedad. La vitrificación o congelación ultrarápida, es un método que evita la formación de cristales de hielo, con lo que el riesgo de que los óvulos se dañen es menor.

\subsection{Criopreservación de embriones}

Consiste en congelar embriones una vez han sido fecundados. Es un método de preservación de fertilidad establecido, usado ampliamente como tratamiento de infertilidad. Requiere también del periodo y tratamiento de estimulación ovárica con inyecciones diarias de hormonas estimulantes de folículos y posterior recolección de ovocitos por aspiración transvaginal y bajo sedación. Posteriormente, se procede a la congelación lenta o vitrificación (evita la formación de cristales intracelulares), técnica con la que se han reportado tasas de gestación de parto de alrededor del $40 \%$.Como se ha mencionado antes, en el caso de pacientes con tumores hormonosensibles, se puede optar por la estimulación ovárica con letrozol o tamoxifeno (asociado no a la administración de FSH).

\subsection{Criopreservación de tejido ovárico}

Presenta varias ventajas respecto a las dos anteriores, no retrasa el inicio del tratamiento ya que no requiere estimulación ovárica, no requiere que la paciente tenga pareja, se puede aplicar en los casos en los que está contraindicada la hiperestimulación ovárica y puede ser aplicado tanto a la mujer adulta, como a las pacientes prepúberes. Consiste en obtener tejido ovárico por vía laparoscópica, extraerlo para su criopreservación y posteriormente reimplantarlo. Hay que tener en cuenta que el tejido extirpado sufre y con ello se pierden alrededor entre un $30-50 \%$ de los folículos primordiales extirpados. Por ello, la efectividad de esta técnica es aún más incierta en mujeres mayores de 40 años donde la reserva de folículos primordiales es menor. La media de duración del implante es de alrededor de 4-5 años. Posteriormente el tejido extirpado se puede implantar en la pelvis (permitiendo una concepción natural) o en otras áreas de forma subcutánea para que ejerza su función endocrina (de secreción hormonal), función que recupera al cabo de unos meses.

Las desventajas son que exige una intervención quirúrgica, que es aún experimental con pocas gestaciones publicadas y que se desconoce la magnitud del riesgo de reimplantar junto con el tejido ovárico célu- las malignas (Meirow et al., 2008; Sanchez-Serrano et al., 2009). En cuanto al riesgo de reimplantación de células malignas, en pacientes con cánceres en estadios iniciales, sin evidencia de enfermedad a distancia, el riesgo parece bajo y por el momento no hay evidencia de recaída en las pacientes que se han sometido a este proceso.Es una técnica aún muy reciente, considerada experimental, aunque ya se han reportado más de 30 casos de embarazos a término satisfactorios (Callejo et al., 2013; Donnez et al., 2013).

\subsection{Transposición ovárica}

También llamada ooforepexia. Consiste en, mediante cirugía, desplazar los ovarios lo más lejos posible del campo de irradiación y fijarlos a la pared pélvica. Es eficaz en preservar la función ovárica en un $50 \%$. Los ovarios se verán afectados además de por la irradiación que llegue a la pared pélvica, por la alteración de la vascularización del ovario. Tras el tratamiento se ha descrito casos de embarazos sin necesidad de volver a colocar el ovario, aunque si es preciso, mediante nueva intervención quirúrgica se recolocan en la pelvis.

\subsection{Cirugía ginecológica conservadora}

Según el tipo de cáncer ginecológico, se puede practicar una cirugía menos radical en la medida de lo posible para intentar preservar la fertilidad. Es el caso de la traquelectomía: cirugía conservadora en pacientes con estadio inicial de carcinoma de cérvix en la que se extirpa de forma total el cérvix, pero se conservan el útero y los ovarios.

\section{CONSIDERACIONES ESPECIALES}

Hay otras consideraciones de interés en relación con el tema de este capítulo. Una de ellas es el caso de las mujeres con antecedentes de cáncer de mama hormonosensible que buscan una gestación tras finalizar los tratamientos sometiéndose o no a tratamientos de fertilidad. No se ha demostrado que una gestación tras el cáncer de mama empeore el pronóstico de estas pacientes. Se aconseja esperar al menos 6 meses tras finalizar tanto la quimioterapia como la administración de tamoxifeno.

Históricamente, a las mujeres con cáncer de mama hormonosensible no se les aconsejaba someterse a tratamientos de fertilidad que requirieran estimulación ovárica porque con el uso de análogos de las gonadotropinas se evidencia un aumento considerable en los niveles de estrógeno circulantes (Hic- 
key et al., 2009). Otras técnicas de inducción de la ovulación usan tamoxifeno, que aunque también se asocia a niveles aumentados de estradiol tiene acciones antiestrogénicas en el tejido mamario, y otras usan letrozol (Reddy y Oktay, 2012), un inhibidor de la aromatasa, tratamiento adyuvante de elección en pacientes con cáncer de mama hormonosensible, postmenopáusicas.
La realidad es que menos del $10 \%$ de las mujeres que han pasado un cáncer de mama se quedan embarazadas (Peccatori et al., 2013). De hecho la mitad de las mujeres que han pasado cáncer de mama se quedan embarazadas respecto a las mujeres de su misma edad sin dicho antecedente. Por otra parte, el porcentaje de mujeres que conciben tras haber pasado otro tipo de cánceres (melanoma, cáncer de tiroides, cáncer de cérvix, linfomas) es mayor.

\section{BIBLIOGRAFÍA}

Blumenfeld, Z. (2007). How to preserve fertility in young women exposed to chemotherapy? The role of $\mathrm{GnRH}$ agonist cotreatment in addition to cryopreservation of embrya, oocytes, or ovaries. Oncologist, 12, 9, pp. 1044-1054. DOI: 12/9/1044 [pii]10.1634/theoncologist.12-9-1044.

Callejo, J., Salvador, C., Gonzalez-Nunez, S., Almeida, L., Rodriguez, L., Marques, L. et al. (2013). Live birth in a woman without ovaries after autograft of frozen-thawed ovarian tissue combined with growth factors. Journal of Ovarian Research, 6, 1, pp. 33. DOI: 10.1186/1757-2215-6-33.

Cobo, A., Domingo, J., Perez, S., Crespo, J., Remohi, J. y Pellicer, A. (2008). Vitrification: an effective new approach to oocyte banking and preserving fertility in cancer patients. Clinical and Translational Oncology, 10, 5, pp. 268-273.

Cobo, A., Garcia-Velasco, J. A., Domingo, J., Remohi, J. y Pellicer, A. (2013). Is vitrification of oocytes useful for fertility preservation for age-related fertility decline and in cancer patients? Fertility and Sterility, 99, 6, pp. 1485-1495. DOI: 10.1016/j.fertnstert.2013.02.050.

Del Mastro, L., Boni, L., Michelotti, A., Gamucci, T., Olmeo, N., Gori, S. et al. (2011). Effect of the gonadotropin-releasing hormone analogue triptorelin on the occurrence of chemotherapyinduced early menopause in premenopausal women with breast cancer: a randomized trial. Journal of the American Association, 306, 3, pp. 269-276. DOI: 10.1001/jama.2011.991.

Donnez, J., Dolmans, M. M., Pellicer, A., Diaz-Garcia, C., Sanchez Serrano, M., Schmidt, K. T. et al. (2013). Restoration of ovarian activity and pregnancy after transplantation of cryopreserved ovarian tissue: a review of 60 cases of reimplantation. Fertility and Sterility, 99, 6, pp. 1503-1513. DOI: 10.1016/j. fertnstert.2013.03.030.

Hickey, M., Peate, M., Saunders, C. M. y Friedlander, M. (2009). Breast cancer in young women and its impact on reproductive function. Human Reproduction Update, 15, 3, pp. 323-339. DOI: dmn064 [pii]10.1093/humupd/dmn064.

Kishk, E. A. y Mohammed Ali, M. H. (2013). Effect of a gonadotropin-releasing hormone analogue on cyclophosphamideinduced ovarian toxicity in adult mice. Archives of Gynecology and Obstetrics, 287, 5, pp. 1023-1029. DOI: 10.1007/ s00404-012-2658-y.

Lee, S. J., Schover, L. R., Partridge, A. H., Patrizio, P., Wallace, W. H., Hagerty, K. et al. (2006). American Society of Clinical Oncology recommendations on fertility preservation in cancer patients. Journal of Clinical Oncology, 24, 18, pp. 2917-2931. DOI: JCO.2006.06.5888 [pii]10.1200/JCO.2006.06.5888.

Loren, A. W., Mangu, P. B., Beck, L. N., Brennan, L., Magdalinski, A. J., Partridge, A. H. et al. (2013). Fertility preservation for patients with cancer: American Society of Clinical Oncology clinical practice guideline update. Journal of Clinical Oncology, 31, 19, pp. 2500-2510. DOI: JCO.2013.49.2678 [pii]10.1200/ JCO.2013.49.2678.

Meirow, D., Hardan, I., Dor, J., Fridman, E., Elizur, S., Ra'anani, H. et al. (2008). Searching for evidence of disease and malignant cell contamination in ovarian tissue stored from hematologic cancer patients. Human Reproduction, 23, 5, pp. 10071013. DOI: 10.1093/humrep/den055.

Peccatori, F. A., Azim, H. A., Jr., Orecchia, R., Hoekstra, H. J., Pavlidis, N., Kesic, V. et al. (2013). Cancer, pregnancy and fertility: ESMO Clinical Practice Guidelines for diagnosis, treatment and follow-up. Annals of Oncology, 24 Suppl 6, pp. vi160170. DOI: mdt199 [pii]10.1093/annonc/ mdt199.

Reddy, J. y Oktay, K. (2012). Ovarian stimulation and fertility preservation with the use of aromatase inhibitors in women with breast cancer. Fertility and Sterility, 98, 6, pp. 1363-1369. DOI: 10.1016/j. fertnstert.2012.09.022.

Ruddy, K. J., Guo, H., Barry, W., Dang, C. T., Yardley, D. A., Moy, B. et al. (2015). Chemotherapy-related amenorrhea after adjuvant paclitaxel-trastuzumab (APT trial). Breast Cancer Research and Treatment, 151, 3, pp. 589-596. DOI: 10.1007/s10549-015-3426-z.

Sanchez-Serrano, M., Novella-Maestre, E., Rosello-Sastre, E., Camarasa, N., Teruel, J. y Pellicer, A. (2009). Malignant cells are not found in ovarian cortex from breast cancer patients undergoing ovarian cortex cryopreservation. Human Reproduction, 24, 9, pp. 2238-2243. DOI: 10.1093/humrep/dep196.

Siegel, R., Ma, J., Zou, Z. y Jemal, A. (2014). Cancer statistics, 2014. CA A Cancer Journal for Clinicians, 64, 1, pp. 9-29. DOI: 10.3322/caac.21208.

Sonmezer, M. y Oktay, K. (2006). Fertility preservation in young women undergoing breast cancer therapy. Oncologist, 11, 5, pp. 422-434. DOI: 11/5/422 [pii]10.1634/theoncologist.11-5-422.

Zamah, A. M., Mauro, M. J., Druker, B. J., Oktay, K., Egorin, M. J., Cedars, M. I. et al. (2011). Will imatinib compromise reproductive capacity? Oncologist, 16, 10 , pp. 1422-1427. DOI: 10.1634/theoncologist.2011-0137. 